\title{
CM-P00061558
}

Ref.TH.2043-CERN

\section{BOUNDED PERTURBATIONS OF DYNAMICS}

\author{
D. Buchholz *) \\ CERN - Geneva \\ and
}

J. E. Roberts

UER Expérimentale et Pluridisciplinaire de Luminy et Centre de Physique Théorique, Marseille

\section{A B S T R A C T}

If $\alpha$ and $\alpha^{\prime}$ are one-parameter automorphism groups of a von Neumann algebra $\mathcal{M}, \alpha^{\prime}$ is said to be a bounded perturbation of $\alpha$ if $\left\|\alpha_{t}^{\prime}-\alpha_{t}\right\| \rightarrow 0$ as $t \rightarrow 0$. We give a complete characterization of the bounded perturbations $\alpha^{\prime}$ of $\alpha$. In particular, we show that if $\alpha$ can be implemented by a strongly continuous one-parameter group with self-adjoint generator (Hamiltonian) $H$, then $\alpha^{\prime}$ can be implemented in the same way and the corresponding Hamiltonian $H^{\prime}$ can be chosen to be of the form $\mathrm{H}^{\prime}=\mathrm{VHV}^{-1}+\mathrm{h}$, where $\mathrm{V}$ is a unitary of $\mathcal{N}$ and $h=h^{*} \in \mathcal{H}$.

*) On leave of absence from II. Institut für Theoretische Physik, Universität Hamburg. 


\section{Introduction}

The equilibrium states of an infinite system in quantum statistical mechanics are believed to be characterized by the KMS-condition $[1,2]$. Recently Haag, Kastler and Trych-Pohlmeyer $[3,4]$ have taken the important step of deriving this condition from postulates allowing a direct physical interpretation. The novel ingredient of their approach is the claim that equilibrium states should be stable under small local perturbations of the dynamics. Their result has stimulated us to take a fresh look at the concept of perturbations of dynamics and to ask what is the natural class of gentle perturbations of dynamics within the framework of the algebraic approach to quantum theory.

If one thinks in terms of quantum mechanical perturbation theory, one would perturb dynamics by replacing the total Hamiltonian $H$ of the system by

$$
H^{\prime}=H+\lambda h
$$

where $\mathrm{h}$ is a bounded observable and $\lambda$ a small coupling constant. When dealing with infinite quantum systems, the total Hamiltonian has a direct physical significance only in the case of quantum field theory, so that it is important to rewrite the ansatz (1.1) in a form which makes no explicit reference to the total Hamiltonian. The dynamics must be thought of as being described instead by a continuous 1-parameter group $t \rightarrow \alpha_{t}$ of automorphisms of the algebra $\boldsymbol{C}$ of local observables. If $\mathrm{h}$ is a bounded local observable, one can define a perturbed 1parameter group $t \rightarrow \alpha_{t}^{\prime}$ of automorphisms by setting

$$
\alpha_{t}^{\prime}(A)=\Gamma_{t}^{h} \alpha_{t}(A) \Gamma_{t}^{h *}, A \in \theta
$$

where

$$
\Gamma_{t}^{h}=\sum_{n=0}^{\infty} i^{h} \int_{0}^{t} d t_{n} \int_{0}^{t_{n}} d t_{n-1} \cdots \int_{0}^{t_{2}} d t_{1} \alpha_{t_{1}}(h) \alpha_{t_{2}}(h) \cdots \alpha_{t_{n}}(h),
$$

(compare $[5 ;$ Definition 1$]$ and $[6 ;$ Chapter 4$]$ ). It is this notion of perturbed dynamics which was used in deriving the KMS condition in [3]. The connection between (1.1) and (1.2) is as follows: if $\boldsymbol{Q}$ is realized on a Hilbert space $\mathcal{H}$ where $\alpha_{t}(A)=e^{i t A_{A}} e^{-i t H}, A \in \boldsymbol{O}$ for some self-adjoint operator $H^{H}$ on $\mathcal{H}$, then the self-adjoint operator $H^{\prime}=H+h$ satisfies $\alpha_{t}^{\prime}(A)=e^{i t H^{\prime}} A^{-i t H^{\prime}}, \Lambda \in \boldsymbol{C}$.

Another feature of infinite quantum systems is that, in contrast to elementiry quantum mechanics, it is no longer reasonable to idealize the observables of the system as all self-adjoint operators on a Hilbert space and the physical states as density matrices. There are two aspects to this: first of all, in quantum field 
theory the presence of superselection rules makes it necessary to consider inequivalent irreducible representations. Secondly, in statistical mechanics the representations associated with equilibrium states are not irreducible but are typically factorial representations of type III $_{1}$. As a consequence, there are restrictions on the relative Hamiltonian if the perturbed dynamics is again to give a 1-parameter group of automorphisms of the local observable algebra ${ }^{1}$ ). It suffices to suppose that the relative Hamiltonian is itself observable, but it is less clear whether this condition is also necessary.

For these reasons we consider it desirable to have a notion of perturbed dynamics which makes no a priori reference to a relative Hamiltonian, but instead results naturally from the view that dynamics is described by a continuous 1parameter group of automorphisms $t \rightarrow \alpha_{t}$ of the local observable algebra. Such a notion may serve as a useful starting point for a purely algebraic version of perturbation theory. We consider another 1-parameter group of automorphisms $t \rightarrow \alpha_{t}^{\prime}$ to be a "bounded perturbation" of the dynamics if

$$
\left\|\alpha_{t}^{\prime}-\alpha_{t}\right\| \rightarrow 0 \text { as } \quad t \rightarrow 0
$$

It turns out that it is possible to give a rather complete description of bounded perturbations of dynamics; we find in particular that such a perturbation corresponds to a Hamiltonian $\mathrm{H}^{\prime}$ of the form

$$
H^{\prime}=V H V^{-1}+h
$$

where $\mathrm{V}$ is a unitary operator and $\mathrm{h}$ a bounded self-adjoint operator both from the von Neumann algebra of global observables. If the algebra of local observables is a simple $C^{*}$-algebra then $\mathrm{V}$ and $\mathrm{h}$ may even be chosen from the algebra of local observables. The precise results may be found at various points in the text $[$ Theorems $(4.3),(4.8)$, and $(5.4)]$.

\section{Topology}

In our discussion of bounded perturbations of dynamics the local structure of the observable algebra will not play any direct role. In fact, to make things as simple as possible, we shall begin by analysing bounded perturbations of dynamics in the case of a von Neumann algebra Or. In the physical setting $20 \mathcal{L}$ should be thought of as the von Neumann algebra generated by the relevant representation of the observable algebra. In quantum field theory, this would be the direct sum

1) There may well be cases where this condition is too restrictive. For example, if the electromagnetic interaction is considered as a perturbation of strong interaction physics, one would expect the observable algebra to be enlarged by the perturbation so as to include operators measuring the local electric charge. 
over all superselection sectors; in statistical mechanics one might take the cyclic representation generated by an equilibrium state.

We now consider a 1-parameter automorphism group $t \rightarrow \alpha_{t} \in$ Aut of which is weakly continuous in the sense that $\mathbb{R} \ni t \rightarrow \omega \alpha_{t}(A)$ is continuous for each $A \in \boldsymbol{M}$ and each normal state $\omega$ of ofr. Physically $\alpha_{t}$ should be thought of as describing the time evolution of the system.

\subsection{Definition}

A 1-parameter automorphism group $t \rightarrow \alpha_{t}^{\prime} \in$ Aut $M$ is said to be a bounded perturbation of $t \rightarrow \alpha_{t}$ if $\left\|\alpha_{t}^{\prime}-\alpha_{t}\right\| \rightarrow 0$ as $t \rightarrow 0$.

The terminology ${ }^{2}$ is intended to suggest that the relative Hamiltonian of $\alpha^{\prime}$ with respect to $\alpha$ is bounded. In physical language this would mean that a finite energy transfer is involved in passing from $\alpha$ to $\alpha^{\prime}$. If $\alpha_{t}$ were the identity automorphism $\downarrow$ for each $t$, a bounded perturbation of $\alpha$ would just be a norm continuous 1-parameter group $\alpha^{\prime}$ and this corresponds to a bounded generator (Hamiltonian) for $\alpha^{\prime}$ (see, for example, [7; Corollary 4.1.14]).

We set

$$
r_{t}=\alpha_{t}^{\prime} \alpha_{t}^{-1}, t \in \mathbb{R}
$$

and see that

$$
\gamma_{s+t}=\gamma_{s}{ }^{s} \gamma_{t}, \quad s, t \in \mathbb{R}
$$

where if $\beta$ is an automorphism then $s_{\beta}=\alpha_{s} \beta \alpha_{-s}$. Equation (2.2) is the cocycle identity; it says that $\gamma$ is a 1 -cocycle over $\mathbb{R}$ with values in Aut $\boldsymbol{M}$, where Aut $00 t$ is considered as a group carrying an action $\beta \rightarrow s_{\beta}$ of $\mathbb{R}$.

\subsection{Proposition}

$\alpha^{\prime}$ is a bounded perturbation of $\alpha$ if and only if $\gamma$ is a norm continuous 1 -cocycle with values in In $\partial \mathcal{L}$, the group of inner automorphisms of $\mathscr{H}$.

Proof $\left\|\alpha_{t}^{\prime}-\alpha_{t}\right\|=\left\|\gamma_{t}-\iota\right\|$, so $\gamma_{t}$ is norm continuous at $t=0$. However, the cocycle identity (2.2) then shows that $\gamma_{t}$ is norm continuous at $t=s$. Now, by a result of Kadison and Ringrose [8; Theorem 7], any automorphism $\beta$ with $\|\beta-\imath\|<2$ is inner. Hence $\gamma_{t}$ is inner for $t$ in some neighbourhood of 0 . The cocycle identity (2.2) now implies that $\gamma_{t}$ is an inner automorphism for all t. Conversely, if $\gamma_{t}$ is a norm continuous 1-cocycle with values in In Mr, then $\alpha_{t}^{\prime}=\gamma_{t} \alpha_{t}$ is easily seen to be a bounded perturbation of $\alpha . \square$

2) The term norm continuous perturbation is more accurate from a mathematical point of view. 
If $\boldsymbol{L}$ is a von Neumann algebra, we denote by $\mathcal{U}(\boldsymbol{\partial})$ the unitary group of $\boldsymbol{\partial \mathcal { L }}$ in the norm topology, and if $U \in U$ ( $\left(\gamma_{0}\right)$, we denote by ad $U$ the inner automorphism generated by $U$. Let $z$ denote the centre of $\mathcal{M}$, then In $\mathscr{M}$ as a group is just the quotient group of $U(\boldsymbol{M})$ by the central subgroup $U(\boldsymbol{z})$. The next step in the analysis is to see how the norm topology on In $M$ relates to the quotient topology on $U(\boldsymbol{M}) / U(z)$. The norm topology on In $M$ is defined by a metric $\delta$,

$$
\delta\left(\sigma, \sigma^{\prime}\right)=\left\|\sigma-\sigma^{\prime}\right\|=\sup \left\{\left\|\left(\sigma-\sigma^{\prime}\right)(A)\right\|: A \in \operatorname{rr},\|A\| \leq 1\right\} .
$$

The topology on In $\boldsymbol{M}$ induced by the quotient topology on $U(\mathcal{L}) / \mathcal{U}(\boldsymbol{z})$ is defined by a metric $\delta^{\prime}$

$$
\delta^{\prime}\left(\sigma, \sigma^{\prime}\right)=\inf \left\{\left\|u-u^{\prime}\right\|: u, u^{\prime} \in \operatorname{\gamma r}, \sigma=\operatorname{ad} u, \sigma^{\prime}=\operatorname{ad} u^{\prime}\right\} .
$$

\subsection{Proposition}

The metrics $\delta$ and $\delta^{\prime}$ are equivalent so that In $8 \boldsymbol{f}_{\boldsymbol{L}}$ and $\boldsymbol{u}(\boldsymbol{\gamma} / \mathbf{l}) / \boldsymbol{U}(\boldsymbol{z})$ may be identified as topological groups.

Proof We shall show that

$$
\delta^{\prime}\left(\sigma, \sigma^{\prime}\right) \leq \delta\left(\sigma, \sigma^{\prime}\right) \leq 2 \delta^{\prime}\left(\sigma, \sigma^{\prime}\right), \sigma, \sigma^{\prime} \in \operatorname{In} \partial \boldsymbol{\partial r} \text {. }
$$

It suffices to take $\sigma^{\prime}=\iota$ in (2.5). Now

$$
\left\|U A U^{-1}-A\right\|=\|U A-A U\| \leq 2\|A\|\|U-I\|
$$

so

$$
\delta(\sigma, L) \leq 2 \delta^{\prime}(\sigma, L) \text {. }
$$

However, the inequality the other way round is not so easy and we may deduce it from [8; Lemma 5]. But for completeness we give a proof with the aid of two 1emmas. $\square$

\subsection{Lemma}

If $\sigma=\operatorname{ad} U$ then

$$
\delta^{\prime}(\sigma, L)=\inf _{c \in \varphi} \sup _{E \in C} \inf _{\lambda \in \mathbb{Z}}\|\lambda U E-E\|
$$

where $\boldsymbol{\ell}$ denotes the set of finite partitions of the identiy in $\boldsymbol{z}$, i.e. the set of finite sets of mutually orthogonal non-zero projections of $\boldsymbol{z}$ of sum $I$, and $\pi$ the set of complex numbers of modulus 1 .

Proof Given $\mathrm{C}, \boldsymbol{\varphi}$, we may find some $\mathrm{E} \rightarrow \lambda_{\mathrm{E}}, \mathbb{U}, \mathrm{E} \in \mathrm{C}$ with

$$
\sup _{E \in C} \inf _{\lambda \in T}\|E-\lambda U E\|=\sup _{E \in C}\left\|E-\lambda_{E} U E\right\|=\left\|I-\sum_{E \in C} \lambda_{E} E U\right\| .
$$

Since $\sum_{E \in C} \lambda_{E} E$ is a unitary of $\boldsymbol{z}$, we see that

$$
\delta^{\prime}(\sigma, L) \leq \inf _{C \in \varphi} \sup _{E \in C} \inf _{\lambda \in \mathbb{T}}\|\lambda U E-E\| .
$$


On the other hand, given $\varepsilon>0$, there exists a $z \in U(z)$ with

$$
\delta^{\prime}(\sigma, l) \geq\|z U-I\|-\varepsilon \text {. }
$$

Now by approximating the spectral resolution of $z$, we may pick $C \in \boldsymbol{\varphi}$ and $E \rightarrow \lambda_{E} \in \mathbb{T}$ for $E \in C$ with

$$
\left\|z-\sum_{E \in C} \lambda_{E} E\right\|<\varepsilon .
$$

\section{$\|z u-I\|+\varepsilon \geq\left\|\sum_{E \in C} \lambda_{E} E U-I\right\|=\sup _{E \in C}\left\|\lambda_{E} E U-E\right\| \geq \inf _{C \in \varphi} \sup _{E \in C} \inf \|E-\lambda u E\|$.}

Since $\varepsilon$ is arbitrary, (2.6) follows.

\subsection{Lemma}

$$
\delta^{\prime}(\sigma, \iota) \leq \delta(\sigma, \iota)
$$

Proof Pick $U \in \mathcal{U}(\boldsymbol{M})$ with $\sigma=$ ad $U$. Given $\varepsilon>0$, dissect $\mathbb{T}$ into intervals $\Delta_{1}, \ldots, \Delta_{n}$ such that

$$
\left\|(u-\mu) E\left(\Delta_{j}\right)\right\|<\varepsilon, \quad \mu \in \Delta_{j}, \quad j=1,2, \ldots, n
$$

where $E\left(\Delta_{j}\right) \in$ Of denotes the spectral projection of $u$ corresponding to $\Delta_{j}$. Let $F\left(\Delta_{j}\right) \in \boldsymbol{z}$ denote the central support of $E\left(\Delta_{j}\right)$. We may then pick $C \in \boldsymbol{\ell}$ such that if $E \in C$ and $1 \leq j \leq n$, either $E \leq F\left(\Delta_{j}\right)$ or $E F\left(\Delta_{j}\right)=0$. Now let $\lambda_{1}, \lambda_{2}$ be in the spectrum of UE in the reduced von Neumann algebra $\boldsymbol{H}_{E}$. Choose $j_{1}$ and $j_{2}$ such that $\lambda_{1} \in \Delta_{j_{1}}, \lambda_{2} \in \Delta_{j_{2}}$ and $E \leq F\left(\Delta_{j_{1}}\right) F\left(\Delta_{j_{2}}\right)$. Then $F\left(\Delta_{j_{1}}\right) F\left(\Delta_{j_{2}}\right) \neq 0$ so there is a nonzero partial isometry $\mathrm{V} \in$ Ool with $\mathrm{VV}^{*} \leq \mathrm{E}\left(\Delta_{\mathrm{j}_{1}}\right)$ and $\mathrm{V}^{*} \mathrm{~V} \leq \mathrm{E}\left(\Delta_{\mathrm{j}_{2}}\right)$. Now

$$
\begin{aligned}
\left|\lambda_{1}-\lambda_{2}\right| & =\left\|\lambda_{1} v-\lambda_{2} v\right\| \leq\left\|\lambda_{1} v-u v\right\|+\|u v-v u\|+\left\|v u-\lambda_{2} v\right\| \\
& \leq\left\|\left(\lambda_{1}-u\right) E\left(\Delta_{j_{1}}\right) v\right\|+\|G-\imath\|+\left\|v E\left(\Delta_{j_{2}}\right)\left(u-\lambda_{2}\right)\right\| \\
& \leq 2 \varepsilon+\|\sigma-\iota\| .
\end{aligned}
$$

Thus

$$
\left\|\lambda_{1} E-U E\right\| \leq 2 \varepsilon+\|G-l\| \text {. }
$$

Hence

$$
\sup _{E \in C} \inf _{\lambda \in \mathbb{T}}\|E-\lambda U E\| \leq 2 \varepsilon+\|G-\iota\| \text {. }
$$

Lemma 2.4 now gives $\delta^{\prime}(\sigma, \iota) \leq \delta(\sigma, \iota)+2 \varepsilon$, and since $\varepsilon$ is arbitrary, we have completed the proof of the lemma, which in turn completes the proof of Proposition 2.3.

Remark If we combine the techniques of Lemma 2.5 with $[9$; Lemma $2.3 .10 a]$, it is easy to derive a formula for the spectrum of an inner automorphism in the Banach algebra $B(\boldsymbol{O})$ of weakly continuous linear operators from $\boldsymbol{M}$ into $\boldsymbol{H}$. Since this formula is nowhere needed here we state it without proof.

$$
\left.S_{p} \text { ad } U=\bigcap_{C \in \varphi \in \in C} U \lambda_{1} \lambda_{2}^{-1}: \lambda_{1}, \lambda_{2} \in S_{P} m_{E} U E\right\}
$$


Our goal in the remainder of this section is to show that we can find a continuous mapping $t \rightarrow U_{t} \in \mathcal{U}(\boldsymbol{r})$ such that $\gamma_{t}=$ ad $U_{t}$. Of course, if one could find a continuous cross-section s: In $80 U \rightarrow U(M)$, i.e. ados is the identity on In rro, then we could set $U_{t}=s\left(\gamma_{t}\right)$. However, this is much too optimistic because there is no such cross-section even when $M$ is the $2 \times 2$ matrix algebra. Instead, we shall show that we can define $s$ on some neighbourhood of the identity in In $\mathscr{C}$ so that it is a continuous local cross-section and this will suffice to lift $\gamma$ to $U(O)$. We state the result in more generality than needed because we feel that the following proposition is of interest in its own right.

\subsection{Proposition}

Let $\mathcal{Q}$ be a $c^{*}$-algebra with identity and $\mathcal{L}$ a $c^{*}$-subalgebra containing the identity. Let $\phi: \boldsymbol{a} \rightarrow \boldsymbol{b}$ be a conditional expectation from $\boldsymbol{a}$ to $\boldsymbol{b}$, i.e. a positive linear mapping with $\phi(I)=I$ satisfying

$$
\phi(B A)=B \phi(A), \quad A \in Q, B \in \mathscr{L} \text {. }
$$

Let $u(\boldsymbol{C})$ and $u(\boldsymbol{b})$ denote the unitary groups of $\boldsymbol{Q}$ and $\mathcal{L}$ in the norm topology so that $U(\mathcal{L})$ is a closed subgroup of $\boldsymbol{U}(\boldsymbol{\alpha})$. Then the homogeneous space $u(Q) / U(\mathcal{L})$ of right cosets admits a local cross-section.

Proof Let $\boldsymbol{V}=\{\boldsymbol{u} \in \boldsymbol{U}(\boldsymbol{a}): \phi(u)$ is invertible $\}$. Since the set of invertible elements of $\mathcal{L}$ is open in the norm topology and $\phi$ is continuous, $\mathcal{V}$ is an open neighbourhood of the identity in $u(\mathcal{Q})$. We see from (2.8) that, if $U \in V$, $|\phi(U)| \phi(U)^{-1} U$ is independent of the choice of $U$ in its right coset. Here $|\phi(U)|$ denotes as usual the positive square root of $\phi(U)^{*} \phi(U)$. Also $|\phi(U)| \phi(U)^{-1} \in \mathcal{U}(\mathscr{L})$, so we get a local cross-section $s$ of the canonical mapping $p$, say, of $u(\alpha)$ onto $u(\theta) / u(\mathcal{})$ : this is defined on the open set $p(\vartheta)$ by setting

$$
\text { s.p }(u)=|\phi(u)| \phi(u)^{-1} u \text {. }
$$

It remains to show that $s$ is continuous or equivalently that sop is continuous. Now $\mathrm{U} \rightarrow \phi(\mathrm{U})$ is norm continuous, $\mathrm{B} \rightarrow \mathrm{B}^{-1}$ and $\mathrm{B} \rightarrow|\mathrm{B}|$ are norm continuous on the set of invertible elements of $\mathscr{L}$ and $\phi$ is bounded on the unit ball of $\boldsymbol{G}$. Hence, $U \rightarrow \operatorname{sop}(U)=|\phi(U)| \phi(U)^{-1} u$ is norm continuous on $\vartheta$.

In the case where $\boldsymbol{Q}$ is $\boldsymbol{B}(\mathcal{H}), \mathcal{L}$ is the complex numbers $\mathbb{C}$ and $\phi$ is a vector state, this method of finding local cross-sections was used by Wigner [10; Chapter 5] in his treatment of representations of the Poincaré group. In more learned terms, the conclusion of Proposition 2.6 is that $U(\sigma)$ is a locally trivial fibre bundle with base $u(q) / U(b)$ and fibre $u(\mathcal{L})$.

Proposition 2.6 may be applied to the case under investigation because, as follows from the results of Kadison and Singer $[11$; Lemmas 2 and 3$]$, there is a conditional expectation from the von Neumann algebra or onto its centre $z$. In fact, we may improve this slightly. 


\section{$2.7 \quad$ Lemma}

If $\boldsymbol{\gamma r}$ is a von Neumann algebra acted on by a weakly continuous 1-parameter group of automorphism $\alpha$ and $\xi$ is the centre of $\mathscr{R}$, then there is a conditional expectation from $\mathscr{H Z}$ to $\boldsymbol{z}$ such that $\alpha_{t} \phi=\phi \alpha_{t}, t \in \mathbb{R}$.

Proof Let $\Phi$ denote the set of bounded linear mappings of $M$ into $\boldsymbol{z}$ with the point-weak-open topology. In this topology the convergence of a net $\left\{\phi_{\beta}\right\} \subset \Phi$ to $\phi$ means that $f \phi_{\alpha}(\mathrm{A})$ converges to $\mathrm{f} \phi(\mathrm{A})$ for all $\mathrm{f} \in \boldsymbol{Z}_{*}$ and $\mathrm{A} \in \boldsymbol{M}$. In this topology, the unit ba11

$$
\Phi_{1}=\left\{\phi \in \Phi: \quad \sup \frac{\|\Phi(A)\|}{\|A\|} \leq 1\right\}
$$

is compact (compare [12]). Now the set of conditional expectations from $\mathscr{H}$ to $\boldsymbol{z}$ is a non-void compact convex set in $\Phi$. Also if $\phi_{t}=\alpha_{t} \phi \alpha_{-t}$ and $\phi$ is a conditional expectation, then so is $\phi_{t}$. Hence, by the Markov-Kakutani theorem (see $[13 ; \mathrm{V} .10 .6])$, we may find a conditional expectation $\phi$ such that $\phi_{t}=\phi$ for al1 $t \in \mathbb{R} . \mathbf{D}$

\subsection{Proposition}

$\alpha^{\prime}$ is a bounded perturbation of $\alpha$ if and only if there is a norm continuous mapping $(s, t) \rightarrow \alpha_{s}\left(U_{t}\right)$ with values in the unitary group of $m$ such that

$$
\alpha_{t}^{\prime}(A)=u_{t} \alpha_{t}(A) u_{t}^{*}, \quad A \in m r \text {. }
$$

Proof Let $\alpha^{\prime}$ be a bounded perturbation of $\alpha$. By Proposition 2.2, $\gamma$ is continuous as a mapping from $\mathbb{R}$ to In $\mathscr{O}$ and by Proposition 2.3, $\gamma$ is still continuous as a mapping from $\mathbb{R}$ to $\boldsymbol{U}(\boldsymbol{m}) / \mathcal{U}(\boldsymbol{z})$. Now applying Proposition 2.6 with $\boldsymbol{U}=\boldsymbol{m}$, $\mathcal{L}=\xi$ and $\phi$ a conditional expectation such that $\phi \alpha_{t}=\alpha_{t} \phi$ (Lemma 2.7), we can find a local cross-section of $\boldsymbol{u}(\boldsymbol{z}) \rightarrow \boldsymbol{u}(\boldsymbol{\partial t}) / \boldsymbol{u}(\boldsymbol{z})$ which commutes with the action of $\mathbb{R}$. Since $(s, t) \rightarrow{ }^{s} \gamma_{t}$ is norm continuous, we can find a continuous mapping $(s, t) \rightarrow \alpha_{s}\left(U_{t}\right)$ from $\mathbb{R} \times[-\varepsilon, \varepsilon]$ to $\boldsymbol{U}(\mathscr{O})$ for some $\varepsilon>0$, such that $\gamma_{t}=$ ad $U_{t}$ and $U_{0}=I$. Thus we have satisfied (2.9) for $t \in[-\varepsilon, \varepsilon]$. We now extend $U_{t}$ to $[-2 \varepsilon, 2 \varepsilon]$ by setting

$$
u_{t+\varepsilon}=u_{\varepsilon} \alpha_{\varepsilon}\left(u_{t}\right), u_{-t-\varepsilon}=u_{-\varepsilon} \alpha_{-\varepsilon}\left(u_{-t}\right), \quad t \in[0, \varepsilon] \text {. }
$$

It is clear that $U_{t}$ so extended is continuous and satisfies (2.9) for $t \in[-2 \varepsilon, 2 \varepsilon]$. Using this process iteratively, we can define a norm continuous mapping $(s, t) \rightarrow \alpha_{s}\left(U_{t}\right)$ satisfying (2.9). The converse statement is trivial. $\mathbf{\square}$

In the above proof we have used the fact that $\gamma$ is a cocycle when extending $t \rightarrow U_{t}$ with the help of (2.10). However, using methods from the theory of fibre bundles, sharper results can be proved; for example, any continuous mapping from a contractible space into In $\mathscr{O C}$ may be 1 ifted to a continuous mapping into $U$ ( $)$ ). 


\section{Cohomology}

This section is devoted to some rather standard cohomological considerations ${ }^{3}$ ). We begin by giving the formal definition of the cohomology we shall be using. Since we have been working in the norm topology but $t \rightarrow \alpha_{t}$ is only assumed to be weakly continuous, it is convenient to introduce the weakly dense $C^{*}$-algebra $\boldsymbol{r r}_{0}=\left\{\mathrm{A} \in \boldsymbol{r r}:\left\|\alpha_{\alpha_{t}}(\mathrm{~A})-\mathrm{A}\right\| \rightarrow 0\right.$ as $\left.t \rightarrow 0\right\}$ consisting of those elements of which have norm continuous orbits under $\alpha$. We let $z_{0}=\boldsymbol{m} \boldsymbol{\tau}_{0} \cap z_{;} ; \alpha_{t}$ and $\alpha_{t}^{\prime}$ induce automorphisms of $\boldsymbol{z}_{0}$ and $\boldsymbol{z}_{0}$.

A $\underline{1-\text { cocycle }} \Gamma \in z_{\alpha}^{1}\left(\mathbb{R}, U\left(\pi_{0}\right)\right)$ is a continuous mapping $\Gamma: \mathbb{R} \rightarrow U\left(\boldsymbol{m}_{0}\right)$ such that $\Gamma_{0}=I$ and

$$
\Gamma_{s} \alpha_{s}\left(\Gamma_{t}\right)=\Gamma_{s+t}
$$

A 1 -cocycle $\Gamma$ of the form $\Gamma_{t}=U \alpha_{t}(u)^{-1}$ with $u \in U\left(\boldsymbol{m}_{0}\right)$ is called a 1-coboundary. Two 1-cocycles $\Gamma, \Gamma^{\prime}$ are said to be equivalent or to differ by a 1 -coboundary if there exists a $u \in \mathcal{U}\left(\boldsymbol{m}_{0}\right)$ such that

$$
\Gamma_{t}^{\prime}=u^{-1} \Gamma_{t} \alpha_{t}(u)
$$

The equivalence class of $\Gamma$ is called the cohomology class of $\Gamma$ and the set of cohomology classes is denoted by $H_{\alpha}^{1}\left(R, U\left(\boldsymbol{\sim} t_{0}\right)\right)$. We can of course replace $U\left(\boldsymbol{m \tau _ { 0 }}\right)$ in these definitions by another topological group on which $\mathbf{R}$ acts continuously by automorphisms. In particular, replacing $U\left(20 \tau_{0}\right)$ by In $2 \mathcal{L}_{0}$ with the norm topology, the 1 -cocycle $\gamma$ of $(2.1)$ is an element of $z_{\alpha}^{1}\left(\mathbb{R}, \operatorname{In} \alpha \mathcal{L}_{0}\right)$.

We also need the definition of the second cohomology of $\mathbf{R}$ with coefficients in the Abelian group $\mathcal{U}\left(\boldsymbol{z}_{0}\right)$. A 2 -cocycle $z \in \mathbb{z}_{\alpha}^{2}\left(\mathbb{R}, \boldsymbol{U}\left(\boldsymbol{z}_{0}\right)\right)$ is a continuous mapping $z: \mathbb{R} \times \mathbb{R} \rightarrow U\left(\xi_{0}\right)$ such that $z(0, s)=z(s, 0)=I, s \in \mathbb{R}$ and

$$
z(s, t) z(s+t, u)=\alpha_{s}(z(t, u)) z(s, t+u) .
$$

A 2-cocycle $z$ of the form $z(s, t)=\lambda_{s+t} \lambda_{s}^{-1} \alpha_{s}\left(\lambda_{t}^{-1}\right)$ for some continuous mapping $\lambda$ : $\mathbb{R}+u\left(\xi_{0}\right)$ is called a 2-coboundary. Two 2-cocycles are said to be equivalent or to differ by a 2 -coboundary if there is a continuous mapping $\lambda: \mathbb{R} \rightarrow \mathcal{U}\left(\boldsymbol{z}_{0}\right)$ such that

$$
z^{\prime}(s, t)=\lambda_{s+t}^{-1} z(s, t) \lambda_{s} \alpha_{s}\left(\lambda_{t}\right)
$$

The equivalence class of $z$ is called the cohomology class of $z$ and the set of cohomology classes is denoted by $\mathrm{H}_{\alpha}^{2}\left(\mathbb{R}, \boldsymbol{u}\left(\boldsymbol{z}_{0}\right)\right)$.

3) The reader with no appetite for these questions may omit this section and proceed by assuming that the mapping $u$ of Proposition 2.8 satisfies the identity $(3.1)$. 
We have seen in Proposition 2.8 that if $\alpha^{\prime}$ is a bounded perturbation of $\alpha$ then there is a continuous mapping $U: \mathbb{R} \rightarrow U\left(\boldsymbol{O t}_{0}\right)$ such that

$$
\alpha_{t}^{\prime}=\operatorname{ad} u_{t} \alpha_{t} \text {. }
$$

In this section we show that we may choose $U$ in such a way that it satisfies the cocycle identity (3.1). To this end we introduce a function $z$ of two variables which measures the deviation of $u$ from a 1 -cocycle

$$
z(s, t)=u_{s} \alpha_{s}\left(u_{t}\right) u_{s+t}^{-1}
$$

and whose properties are summed up in

\subsection{Proposition}

$z$ is a 2 -cocycle with values in $u\left(\xi_{0}\right), z \in z_{\alpha}^{2}\left(\mathbb{R}, U\left(z_{0}\right)\right)$. The cohomology class of $z$ depends only on the cohomology class of $\gamma \in Z_{\alpha}^{1}\left(\mathbb{R}\right.$, In $\left.\gamma \boldsymbol{r}_{0}\right)$. Hence there is a 1 -cocycle $\Gamma \in Z_{\alpha}^{1}\left(\mathbb{R}, U\left(20 \tau_{0}\right)\right)$ satisfying (3.5) if and only if $z$ is a 2-coboundary.

Proof The proof is a routine computation. The unitary $z(s, t)$ defined in (3.6) must 1 ie in $z_{0}$ since it induces the trivial automorphism and has a norm continuous orbit under $\alpha$. It is clear that $z: \mathbb{R} \times \mathbb{R} \rightarrow U\left(z_{0}\right)$ is continuous. Now

$$
z(s, t) z(s+t, u)=u_{s} \alpha_{s}\left(u_{t} \alpha_{t}\left(u_{u}\right)\right) u_{s+t+u}^{-1}=\alpha_{s}(z(t, u)) u_{s} \alpha_{s}\left(u_{t+u}\right) u_{s+t+u}^{-1}
$$

where we have used the fact that $\alpha_{s}(z(t, u)) \in \xi$ and thus commutes with $U_{s}$. This proves (3.3), so $z \in Z_{\alpha}^{2}\left(\mathbb{R}, u\left(z_{0}\right)\right)$. Now if $u^{\prime}: \mathbb{R} \rightarrow u\left(z_{0}\right)$ is continuous and satisfies (3.5), then the cocycle $z^{\prime}$ derived from $u^{\prime}$ is related to $z$ by

$$
z^{\prime}(s, t)=\lambda_{s} \alpha_{s}\left(\lambda_{t}\right) z(s, t) \lambda_{s+t}^{-1}
$$

where $\lambda_{s}=u_{s}^{\prime} u_{s}^{-1} \in U\left(z_{0}\right)$. Thus $z^{\prime}$ and $z$ differ by a 2-coboundary and the cohomology class of $z$ depends only on $\gamma \in z_{\alpha}^{1}\left(\mathbb{R}\right.$, In $\left.\boldsymbol{M}_{0}\right)$. However, if $v \in \mathcal{U}\left(\boldsymbol{z}_{0}\right)$ and $\gamma_{s}^{\prime}=\operatorname{adV} \gamma_{s}$ ad $\alpha_{s}(V)^{-1}$, then $\gamma_{s}^{\prime}=\operatorname{ad}\left(V U_{s} \alpha_{s}(V)^{-1}\right)$ and the $z^{\prime}$ computed from (3.6) with $U_{s}^{\prime}=V_{s} \alpha_{s}(V)^{-1}$ in place of $U_{s}$ equals $z$. Hence the cohomology class of $z$ depends only on the cohomology class of $\gamma$.

Another way of looking at this proposition is to say that we have an exact sequence of cohomology

$$
H_{\alpha}^{1}\left(\mathbb{R}, u\left(m_{0}\right)\right) \rightarrow H_{\alpha}^{1}\left(\mathbb{R}, I_{n} m_{0}\right) \rightarrow H_{\alpha}^{2}\left(R, u\left(z_{0}\right)\right)
$$

induced by the exact sequence of topological groups

$$
1 \longrightarrow u(z) \longrightarrow u(g r) \longrightarrow \operatorname{In} \text { or } \longrightarrow 1 \text {. }
$$


Hence to show that there is a $\Gamma \in z_{\alpha}^{1}\left(\mathbb{R}, u\left(\sigma_{0}\right)\right)$ with $\alpha_{t}^{\prime}=\operatorname{ad} \Gamma_{t} \alpha_{t}$, it is enough to know that $H_{\alpha}^{2}\left(\mathbb{R}, \mathcal{U}\left(\boldsymbol{z}_{0}\right)\right)$ is trivial, i.e. that every 2-cocycle is a 2-coboundary.

For completeness we shall give a proof of this, as most readers have probably never seen one, even for the of ten quoted case where $U\left(z_{0}\right)$ is the circle group $T$. The result that $H^{2}(\mathbb{R}, \boldsymbol{T})$ is trivial apparently goes back to Iwasawa who proves [14; Lemma 3.4] that every extension of $\mathbb{T}$ by $\mathbb{R}$ splits. We make no use of the concept of group extension and begin with a lemma which is purely algebraic in nature and holds for any coefficient group.

\subsection{Lemma}

a) If $z$ is a 2 -cocycle such that

$$
z(n, t)=I \quad \text { for } \quad 0 \leq t \leq 1, n \in \mathbb{Z}
$$

then for each $t \in \mathbb{R}, s \rightarrow \alpha_{-s}(z(s, t))$ is periodic with period 1 .

b) If $z(s, t)=I$ for $0 \leq t \leq 1$ then

$$
z(s, t)=I \quad \text { for } \quad s, t \in \mathbb{R} \text {. }
$$

Proof From the cocycle identity in the form

$$
z(n, 1) z(n+1, t)=\alpha_{n}(z(1, t)) z(n, 1+t)
$$

we deduce in case a) by iteration that $z(n, t)=I$ for $t \geq 0$ and $n \in \mathbb{Z}$. From the cocycle identity in the form

$$
z(-n, n)=\alpha_{-n}(z(n,-t)) z(-n, n-t)
$$

we deduce that $z(n,-t)=I$ for $t \leq n \in \mathbb{Z}$. From the cocycle identity in the form

$$
z(n,-1) z(n-1, t)=\alpha_{n}(z(-1, t)) z(n, t-1)
$$

since $\mathbf{z}(n,-1)=I$ for $n \in \mathbb{Z}, n \geq 0$ we deduce by iteration that $\mathbf{z}(n, t)=I$ for $\mathrm{n} \in \mathbb{Z}, \mathrm{n} \geq 0$. However $(*)$ now tells us that $\mathbf{z}(-\mathrm{n}, \mathrm{n}-\mathrm{t})=\mathrm{I}$ for $\mathrm{n} \in \mathbb{Z}, \mathrm{n} 20$. Hence $\mathbf{z}(n, t)=I$ for $n \in \mathbb{Z}, t \in \mathbb{R}$. At this stage, if we replace $\mathbb{Z}$ by $\mathbb{R}$ everywhere, we have proved (b). To complete the proof of (a), we simply use the cocycle identity again in the form

$$
z(n, s) z(n+s, t)=\alpha_{n}(z(s, t)) z(n, s+t)
$$

which shows that $z(n+s, t)=\alpha_{n}(z(s, t))$ as required.

The utility of Lemma 3.2 lies in the fact that every cohomology class contains a cocycle satisfying the hypothesis of part (a).

\section{$3.3 \quad$ Lemma}

Let $\mathrm{z}$ be a 2 -cocycle and define $\lambda_{0}=\mathrm{I}$,

$$
\lambda_{t+n}=\lambda_{n} z(n, t), 0 \leq t<1, n \in \mathbb{Z}
$$

then $t \rightarrow \lambda_{t}$ is continuous and the 2 -cocycle $z^{\prime}$ defined by

$$
z^{\prime}(s, t)=\lambda_{s} \alpha_{s}\left(\lambda_{t}\right) z(s, t) \lambda_{s+t}^{-1}
$$


satisfies $\mathbf{z}^{\prime}(n, t)=I$ for $0 \leq t \leq 1$ and $n \in \mathbb{Z}$. If $\|z(s, t)-I\| \leq \delta$ for $0 \leq s, t \leq 1$ then $\|z(s, t)-I\| \leq 2 \delta$, for $0 \leq s, t \leq 1$.

Proof Let $n \in \mathbb{Z}$, then since $t \rightarrow z(n, t)$ is continuous and $z(n, 0)=I$, it is clear that $t \rightarrow \lambda_{t}$ is continuous. Now $z^{\prime}(n, t)=\lambda_{n} \alpha_{n}\left(\lambda_{t}\right) \lambda_{n}^{-1}$, but $\lambda_{t}=I$ for $0 \leq t \leq 1$, so $s^{\prime}(n, t)=I$ for $0 \leq t \leq 1$. If $0 \leq s, t \leq 1$ and $s+t \leq 1$, then $z^{\prime}(s, t)=z(s, t)$. If $0 \leq s, t \leq 1$ but $s+t \leq 1$, then $\lambda_{s+t}=z(1, s+t-1)$ and $z^{\prime}(s, t)=z(s, t) z(1, s+t-1)^{-1}$. Hence $\|z(s, t)-I\|<\delta$ for $0 \leq s, t \leq 1$ implies $\left\|z^{\prime}(s, t)-I\right\|<2 \delta$ for $0 \leq s, t \leq 1$.

\subsection{Corollary}

A 2 -cocycle $\mathrm{z}$ with $\mathrm{z}(\mathrm{s}, \mathrm{t})=\mathrm{I}$ for $0 \leq s, t \leq 1$ is a 2 -coboundary.

Proof By Lemma 3.3 we may suppose further that $z(n, t)=I$ for $0 \leq t \leq 1$ and $\mathrm{n} \in \mathbb{Z}$, and hence by Lemma $3.2 \mathrm{a} s \rightarrow \alpha_{-s}(z(s, t))$ is periodic with period 1 . But then $z(s, t)=I$ for $0 \leq t \leq 1, s \in \mathbb{R}$ and the result follows from Lemma 3.2b.

Remark If $z$ is a 2 -cocycle and $\mu \in \mathbb{R}$, then $z^{\prime}(s, t)=z(\mu s, \mu t)$ is a 2 -cocycle for the action $t \rightarrow \alpha_{\mu t}$. This allows us to scale the results in Lemmas 3.2 and 3.3 and Corollary 3.4 and we shall make free use of this in proving the next result.

\subsection{Proposition}

If $\boldsymbol{Q}$ is an Abelian $C^{*}$-algebra and $t \rightarrow \alpha_{t}$ is a strongly continuous 1-parameter group of automorphisms of $\boldsymbol{Q}$, then $\mathrm{H}_{\alpha}^{2}(\mathbb{R}, u(Q))$ is trivial.

Proof Let $z \in Z_{\alpha}^{2}(\mathbb{R}, U(Q))$ and $0<\mathrm{k}<1 / \sqrt{2}$, then we may pick $\varepsilon>0$ such that $\|z(s, t)-I\| \leq k$ for $0 \leq s, t \leq \varepsilon$. By Lemma 3.3 we may find an equivalent 2-cocycle $z^{\prime}$ such that $\left\|z^{\prime}(s, t)-I\right\| \leq 2 k$ for $0 \leq s, t \leq \varepsilon$ and $z^{\prime}(n \varepsilon, t)=I$ for $0 \leq t \leq \varepsilon$ and $n \in \mathbb{Z}$. By Lemma 3.2a, $s \rightarrow \alpha_{-s}\left(z^{\prime}(s, t)\right)$ is periodic with period $\varepsilon$. In particular, $\left\|z^{\prime}(s, t)-I\right\| \leq 2 k$ for $s \in \mathbb{R}$ and $0 \leq t \leq \varepsilon$. Now let $1 \circ$ g denote the principal value of the logarithm defined for operators $A \in \mathcal{G}$ with spectrum in the complex plane cut along the negative real axis. The spectrum of $g^{\prime}(s, t)$ for $0 \leq t \leq \varepsilon$ is contained in $\{\lambda \in \mathbb{C}:|\lambda|=1$, Re $\lambda>0\}$. Hence putting $y(s, t)=\log z^{\prime}(s, t)$ for $0 \leq t \leq \varepsilon$, the cocycle identity gives

$$
y(s, t)+y(s+t, u)=\alpha_{s}(y(t, u))+y(s, t+u)
$$

if $0 \leq t, u, t+u \leq \varepsilon$. Furthermore, $y(s, t)$ is jointly continuous in both variables. Define

$$
c_{t}=-\varepsilon-1 \int_{0}^{\varepsilon} d s \alpha_{-s}(y(s, t))
$$

then $t \rightarrow c_{t}$ is continuous. Also, since $s \rightarrow \alpha+\alpha(y(s, t)$ has period $\varepsilon$,

$$
c_{t}=-\varepsilon^{-1} \int_{u}^{\varepsilon+u} d s \alpha_{-s}(y(s, t))
$$

for all $u \in \mathbb{R}$. Direct computation using (3.7) now shows that

$$
c_{s+t}-\alpha_{s}\left(c_{t}\right)-c_{s}=y(s, t), \text { if } 0 \leq s, t, s+t \leq \varepsilon .
$$


Exponentiating and setting $\lambda_{s}=\exp c_{s}$ we get

$$
\lambda_{s+t} \alpha_{s}\left(\lambda_{t}^{-1}\right) \lambda_{s}^{-1}=z^{\prime}(s, t) \text { if } 0 \leq s, t, s+t \leq \varepsilon .
$$

Since $s \rightarrow \lambda_{s}$, which is defined in $[0, \varepsilon]$, may be extended to a continuous function on all of $\mathbb{R}^{\prime}$, we see from Corollary 3.4 that $z^{\prime}$ is a 2 -coboundary. This completes the proof.

\section{Smoothing}

The results of the previous two sections may be summed up in the following proposition.

\subsection{Proposition}

$\alpha^{\prime}$ is a bounded perturbation of $\alpha$ if and only if there is a strongly continuous 1 -cocycle $\Gamma$ with values in $\mathcal{U}\left(\boldsymbol{\gamma} \boldsymbol{\tau}_{0}\right)$ such that

$$
\alpha_{t}^{\prime}=\operatorname{ad} \Gamma_{t} \alpha_{t}
$$

Our next task is to gain some insight into the set of 1 -cocycles $Z_{\alpha}^{1}\left(\mathbb{R}, U\left(20 \zeta_{0}\right)\right)$. It turns out that differentiable cocycles can be characterized in terms of their derivatives at $t=0$ and we begin with a smoothing procedure ${ }^{4}$ ) which allows us to approximate continuous cocycles by differentiable ones. It is convenient to formulate things in terms of a general $c^{*}$-algebra $\boldsymbol{Q}$.

Let $\boldsymbol{Q}$ be a $c^{*}$-algebra with identity and $\alpha$ a strongly continuous 1-parameter group of automorphisms of $\boldsymbol{Q}$. If $\mathrm{f} \in \mathrm{L}^{1}(\mathbb{R})$ and $\Gamma \in \mathrm{Z}_{\alpha}^{1}(\mathbb{R}, \boldsymbol{u}(\boldsymbol{\sigma}))$, then we set $\Gamma(f)=\int \Gamma_{t} f(t) d t$ so

$$
\|\Gamma(f)\| \leq\|f\|_{1},
$$

whereas the cocycle identity (3.1) gives

$$
\Gamma_{s} \alpha_{s}\left(\Gamma_{f}\right)=\Gamma\left(f_{s}\right) \text {, where } f_{s}(t)=f(t-s)
$$

It follows that $s \rightarrow \Gamma_{s} \alpha_{s}(\Gamma(f))$ is norm differentiable if $s \rightarrow f_{s}$ is norm differentiable in $L^{1}(\mathbb{R})$. Clearly by taking $f$ to be a smooth approximation to the $\delta$ function, we get an operator $\Omega \in \boldsymbol{O}$ as close in norm to the identity as we wish, such that $s \rightarrow \Gamma_{s} \alpha_{s}(\Omega)$ is as smooth as we wish. For our purpose, it suffices to proceed as follows: pick $0<k<1$ and $\tau>0$ such that $\left\|\Gamma_{t}-I\right\| \leq k$ for $0 \leq t \leq \tau$ and set $\Omega=\tau^{-1} \cdot \int_{0}^{\tau} \Gamma_{t} d t$. Then $t \rightarrow \Gamma_{t} \alpha_{t}(\Omega)$ is differentiable in norm and $\|\Omega-I\| \leq \mathrm{k}<1$ so that $\Omega$ is invertible.

4) Smoothing procedures for cocycles are well known in other contexts (see e.g. $[14]$ ). The reader may find the analogy between a 1-cocycle and a 1-parameter unitary group helpful here. 


\subsection{Lemma}

There is a unitary $V \in U(\theta)$ such that $t+\Gamma_{t} \alpha_{t}(V)$ is differentiable in norm. Proof Let $\Omega$ be as above, then $t \rightarrow \Gamma_{t} \alpha_{t}(\Omega)$ is differentiable in norm. Hence so is $t \rightarrow \alpha_{t}(\Omega \star \Omega)=\alpha_{t}(\Omega){ }^{*} \Gamma_{t}^{*} \Gamma_{t} \alpha_{t}(\Omega)$, and $\Omega^{*} \Omega$ is invertible. Since, by the resolvent functional calculus, $B \rightarrow|B|^{-1}$ is differentiable (even analytic) on an open set containing $\Omega^{*} \Omega, t \rightarrow \alpha_{t}\left(|\Omega|^{-1}\right)=\left|\alpha_{t}(\Omega)\right|^{-1}$ is also differentiable. If $\mathrm{V}=\Omega|\Omega|^{-1}$ then $V$ is unitary and $t \rightarrow \Gamma_{t} \alpha_{t}(V)=\Gamma_{t} \alpha_{t}(\Omega) \alpha_{t}\left(|\Omega|^{-1}\right)$ is differentiable in norm.

At this point we are very close to our main result and, for the sake of the reader who would prefer to be spared some of the more detailed information, we interrupt our discussion of $z_{\alpha}^{1}(\mathbb{R}, U(\boldsymbol{Q}))$ to provide the most easily comprehensible version of this result. We suppose, as we may, that for is realized in some faithful normal representation in which $\alpha$ is implemented by a strongly continuous 1 -parameter group of unitary transformations. We then have the situation described in

\subsection{Theorem}

Let 8 \& be a von Neumann algebra on a Hilbert space $\mathcal{H}$ and $\mathrm{H}$ a self-adjoint operator on $\mathcal{H}$ inducing a 1-parameter group $\alpha$ of automorphisms of $\mathcal{H}$ :

$$
e^{i H t} A e^{-i H t}=\alpha_{t}(A), A \in \operatorname{rr} \text {. }
$$

Then $\alpha^{\prime}$ is a bounded perturbation of $\alpha$ if and only if there is a $v \in U$ (gito) and an $h=h^{*} \in \boldsymbol{J f r}_{0}$ such that $\alpha_{t}^{\prime}(A)=e^{i H^{\prime} t} A e^{-i H^{\prime} t}, A \in \mathcal{M}$, where $H^{\prime}$ is the selfadjoint operator defined on $\mathrm{D}\left(\mathrm{H}^{\prime}\right)=\mathrm{VD}(\mathrm{H})$ by

$$
H^{\prime} \Phi=V H V^{-1} \Phi+h \Phi, \quad \Phi \in D\left(H^{\prime}\right)
$$

Proof If $\alpha^{\prime}$ is a bounded perturbation of $\alpha$, then, by Proposition 4.1, there is a 1-cocycle $\Gamma \in Z_{\alpha}^{1}\left(\mathbb{R}, U\left(m_{0}\right)\right)$ with $\alpha_{t}^{\prime}=$ ad $\Gamma_{t} \alpha_{t}$. By Lemma 4.2 there is a $v \in U\left(g_{0}\right)$ such that $t \rightarrow \Gamma_{t} \alpha_{t}(V)$ is differentiable in norm. Let

$$
h=-\left.i \frac{d}{d t} \Gamma_{t} \alpha_{t}(v) v^{-1}\right|_{t=0}
$$

so that $h=h^{*} \in M_{0}$. We now define $H^{\prime}$ to be the self-adjoint generator of

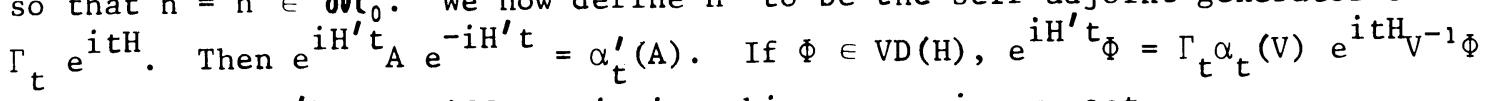
so that $\Phi \in \mathrm{D}\left(\mathrm{H}^{\prime}\right)$ and differentiating this expression we get

\section{$H^{\prime} \Phi=V H V^{-1} \Phi+h \Phi$.}

The symmetry between $\mathrm{H}$ and $\mathrm{H}^{\prime}$ shows that $\mathrm{D}\left(\mathrm{H}^{\prime}\right)=\mathrm{VD}(\mathrm{H})$. Conversely, given $\mathrm{V}$ and $h$, define $H^{\prime}$ by $(4.4)$. It is easy to see that $H^{\prime}$ is self-adjoint. If $h=0$, $e^{i H^{\prime} t}=V e^{i H t} V^{-1}$, so $H^{\prime}$ induces $\alpha_{t}^{\prime}=\operatorname{ad}\left(V \alpha_{t}\left(V^{-1}\right)\right) \alpha_{t}$, which is a bounded perturbation of $\alpha$. Hence we may take $V=I$. Now let $\Gamma_{t}$ be the solution of the differential equation $\frac{d \Gamma_{t}}{d t}=i \Gamma_{t} \alpha_{t}(h)$ such that $\Gamma_{0}=I$, then $\Gamma \in z_{\alpha}^{1}\left(\mathbb{R}, \mathcal{u}\left(z \pi_{0}\right)\right)$ $[6 ;$ chapter 4$]$ and one sees easily that $\Gamma_{t} e^{i H t}=e^{i H^{\prime} t}$. Thus $H^{\prime}$ generates a bounded perturbation of $\alpha . \square$ 
In what follows, we revert to a space-free description, i.e. one making no reference to an underlying Hilbert space. The reader may, however, find it helpful occasionally to think in terms of a Hamiltonian $\mathrm{H}$.

Lemma 4.2 may be rephrased as a statement about cohomology.

\subsection{Corollary}

Every cohomology class of 1 -cocycles in $z_{\alpha}^{1}(\mathbb{R}, u(a))$ contains a representative which is differentiable in norm.

The next step is to parametrize $z_{\alpha}^{1}(\mathbb{R}, u(\sigma))$ as a homogeneous space.

\subsection{Definition}

The inhomogeneous unitary group of a $C^{*}$-algebra $Q$ with identity is defined to be the group, $J U(\theta)$, of pairs $(h, v)$ where $h=h^{*} \in O$ and $v \in U(O)$ with the following composition law:

$$
(h, V)\left(h^{\prime}, V^{\prime}\right)=\left(h+V h^{\prime} V^{-1}, V v^{\prime}\right)
$$

We now define an action of $\mathcal{J} U(\boldsymbol{O})$ on $z_{\alpha}^{1}(\mathbb{R}, u(a))$. If $\Gamma \in z_{\alpha}^{1}(\mathbb{R}, u(\theta))$ and $g=(0, v) \in J U(Q)$, we set

$$
(g \Gamma)_{t}=V \Gamma_{t} \alpha_{t}(V-1)
$$

If $g=(h, I) \in J U(O)$, we proceed as follows: let $\alpha_{t}^{\prime}=\operatorname{ad} \Gamma_{t} \alpha_{t}$ and define $\Gamma^{\prime} \in \mathbb{z}_{\alpha^{\prime}}^{1}(\mathbb{R}, U(Q))$ to be the solution of the differential equation

$$
\frac{d \Gamma_{t}^{\prime}}{d t}=i \Gamma_{t}^{\prime} \alpha_{t}^{\prime}(h)
$$

with the initial condition $\Gamma_{0}^{\prime}=I$. This does define a unique element of $z_{\alpha^{\prime}}^{1}(\mathbb{R}, u(Q))[6 ;$ chapter 3$]$. Then one sets

$$
(g \Gamma)_{t}=\Gamma_{t}^{\prime} \Gamma_{t}
$$

and one verifies immediately that $g \Gamma \in z_{\alpha}^{1}(\mathbb{R}, u(q))$. For $g=(h, v)$ we use the group 1 aw $g=(h, I)(0, V)$ to define $g \Gamma$. A routine computation shows that if $g, g^{\prime} \in J U(Q)$ then $g\left(g^{\prime} \Gamma\right)=\left(g g^{\prime}\right) \Gamma$, so that (4.6) to (4.8) do define an action of $J u(Q)$ on $z_{\alpha}^{1}(\mathbb{R}, u(a))$.

The action of $J U(\alpha)$ on $z_{\alpha}^{1}(\mathbb{R}, u(\alpha))$ induces an action of $J U(\alpha)$ on the set of strongly continuous 1-parameter groups of automorphisms of $\boldsymbol{Q}$. If $\stackrel{\circ}{\Gamma} \in z_{\alpha}^{1}(\mathbb{R}, u(\boldsymbol{Q}))$ denotes the trivial cocycle, $\stackrel{\circ}{\Gamma}_{t}=I, t \in \mathbb{R}$, we define

$$
(g \alpha)_{t}=\operatorname{ad}(g \stackrel{\circ}{\Gamma})_{t} \alpha_{t}, \quad g \in J u(\alpha)
$$

and omit the computation which verifies that this is an action. 
We may also express this action in terms of infinitesimal generators in the sense of semigroup theory on Banach spaces (see, for example, [15]). The infinitesimal generator $\delta$ of $\alpha$ is a norm closed derivation with domain $D(\delta)=\left\{A \in Q: t \rightarrow \alpha_{t}(A)\right.$ is norm differentiable $\}$; if $A \in D(\delta)$ then $\delta(A)=\lim ^{-1}\left(\alpha_{t}(A)-A\right)$. If $g=(h, V) \in J U(Q)$ and $\delta^{\prime}$ is the infinitesimal generator $\stackrel{t \rightarrow 0}{ }$ of $(g \alpha)$, then

$$
\begin{gathered}
D\left(\delta^{\prime}\right)=V D(\delta) V^{-1} \\
\delta^{\prime}(A)=V \delta\left(V^{-1} A V\right) V^{-1}+[i h, A], A \in D\left(\delta^{\prime}\right) .
\end{gathered}
$$

\subsection{Proposition}

$J u(\sigma)$ acts transitively on $z_{\alpha}^{1}(\mathbb{R}, u(\theta))$ and the stability subgroup (isotropy subgroup) of the trivial cocycle $\stackrel{\circ}{\Gamma}$ is the set of pairs (h,V) with $V \in D(\delta)$ and $\delta(V) V^{-1}=i h$, where $\delta$ is the infinitesimal generator of $\alpha$.

Proof Given $\Gamma \in Z_{\alpha}^{1}(\mathbb{R}, u(O))$, pick $V$ as in Lemma 4.2 , let

$$
h=-\left.i \frac{d}{d t} \Gamma_{t} \alpha_{t}(v) v^{-1}\right|_{t=0}
$$

so that $h=h^{*}$ and set $g=(h, v)$. We show that $y U(\mathcal{O})$ acts transitively on $z_{\alpha}^{1}(\mathbb{R}, U(O))$ by showing that $\Gamma=g \Gamma$. Now by $(4.6)$ to $(4.8)(g \Gamma)_{t}=\Gamma_{t}^{\prime} V \alpha_{t}\left(V^{-1}\right)$ where $\Gamma_{t}^{\prime}$ is the solution of the differential equation

$$
\frac{d \Gamma_{t}^{\prime}}{d t}=i \Gamma_{t}^{\prime} V \alpha_{t}\left(V^{-1} h V\right) V^{-1}
$$

with $\Gamma_{0}^{\prime}=I$. On the other hand, if $\hat{\Gamma}_{t}=v^{-1} \Gamma_{t} \alpha_{t}(v)$ then $\hat{\Gamma} \in z_{\alpha}^{1}(\mathbb{R}, u(a))$ satisfies

$$
\frac{d \hat{\Gamma}_{t}}{d t}=i \hat{\Gamma}_{t} \alpha_{t}\left(V^{-1} h V\right)
$$

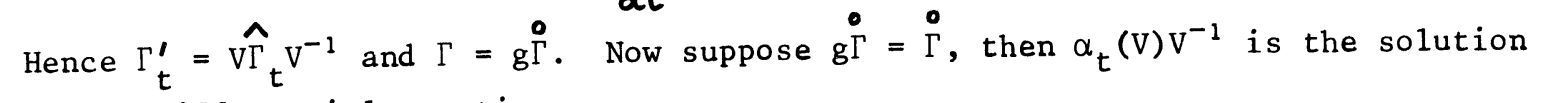
of the differential equation

$$
\frac{d \Gamma_{t}^{\prime}}{d t}=i \Gamma_{t}^{\prime} V \alpha_{t}\left(V^{-1} h V\right) V^{-1}
$$

with $\Gamma_{0}^{\prime}=\mathrm{I}$. Hence $\mathrm{V} \in \mathrm{D}(\delta)$ and $\delta(\mathrm{V}) \mathrm{V}^{-1}=$ ih as required. $\square$

The result above implies that the set of cohomology classes can be characterized as a quotient space of the Hermitian elements, $\boldsymbol{\sigma}_{\mathrm{h}}$ of $\boldsymbol{O}$. We let [Г] denote the cohomology class of $\Gamma \in z_{\alpha}^{1}(\mathbb{R}, U(\theta))$.

\subsection{Proposition}

The mapping $h \rightarrow[(\mathrm{h}, \mathrm{I}) \Gamma]$ maps $\boldsymbol{a}_{\mathrm{h}}$ onto $\mathrm{H}_{\alpha}^{1}(\mathbb{R}, \boldsymbol{u}(\boldsymbol{O}))$ and $[(\mathrm{h}, \mathrm{I}) \Gamma]=\left[\left(\mathrm{h}^{\prime}, \mathrm{I}\right) \Gamma\right]$ if and only if there is a unitary $V \in D(\delta)$ such that $\delta(V)=i\left(V h-h^{\prime} V\right)$. Proof If $\Gamma \in z_{\alpha}^{1}(\mathbb{R}, u(Q))$ then $\Gamma=(h, v) \stackrel{\circ}{\Gamma}$ by Proposition 4.6. But $(\mathrm{h}, \mathrm{V}) \stackrel{\bullet}{\Gamma}=(0, \mathrm{~V})\left(\mathrm{V}^{-1} \mathrm{hV}, \mathrm{I}\right) \stackrel{\circ}{\Gamma}$ so $[\Gamma]=\left[\left(\mathrm{V}^{-1} \mathrm{hV}, \mathrm{I}\right) \stackrel{\circ}{\Gamma}\right]$. Thus $\mathrm{h} \rightarrow[(\mathrm{h}, \mathrm{I}) \stackrel{\circ}{\Gamma}]$ maps $\boldsymbol{G}_{\mathrm{h}}$ onto $\mathrm{H}_{\alpha}^{1}(\mathbb{R}, \mathcal{U}(\boldsymbol{Q}))$. Now $\left[(\mathrm{h}, \mathrm{I}) \Gamma^{\circ}\right]=\left[\left(\mathrm{h}^{\prime}, \mathrm{I}\right) \stackrel{\circ}{\Gamma}^{\circ}\right]$ if, and only if, there is a $\mathrm{v} \in \mathcal{U}(\boldsymbol{Q})$ 
such that $(0, \mathrm{~V})(\mathrm{h}, \mathrm{I}) \stackrel{\circ}{\Gamma}=\left(\mathrm{h}^{\prime}, \mathrm{I}\right) \stackrel{\circ}{\Gamma}$. Thus $\left(\mathrm{VhV}^{-1}-\mathrm{h}^{\prime}, \mathrm{V}\right) \stackrel{\circ}{\Gamma}=\stackrel{\circ}{\Gamma}$, so by Proposition 4.6 $\mathrm{V} \in \mathrm{D}(\delta)$ and $\delta(\mathrm{V}) \mathrm{V}^{-1}=\mathrm{i}\left(\mathrm{VhV}^{-1}-\mathrm{h}^{\prime}\right)$ as required. $\square$

Returning now to bounded perturbations of dynamics, we note that (4.9) may be used to define an action of $\boldsymbol{J} \boldsymbol{U}\left(\boldsymbol{H}_{0}\right)$ on the set of weakly continuous 1-parameter subgroups of automorphisms of Which restrict to give a strongly continuous 1-parameter group of automorphisms of $M_{0}$. By Proposition 4.1, every bounded perturbation $\alpha^{\prime}$ of $\alpha$ is of the form $\alpha_{t}^{\prime}=$ ad $\Gamma_{t} \alpha_{t}$ for some $\Gamma \in z_{\alpha}^{1}\left(\boldsymbol{R}, \boldsymbol{U}\left(\boldsymbol{m}_{0}\right)\right)$ so we obtain the space-free version of our main result.

\subsection{Theorem}

If $\alpha$ and $\alpha^{\prime}$ are weakly continuous 1-parameter groups of automorphisms of $\alpha^{\prime}$ is a bounded perturbation of $\alpha$ if and only if $\alpha^{\prime}$ and $\alpha$ lie on the same orbit under $\boldsymbol{J} \boldsymbol{U}\left(\boldsymbol{M} \boldsymbol{H}_{0}\right)$. The infinitesimal generators $\delta$ and $\delta^{\prime}$ of the restrictions of $\alpha$ and $\alpha^{\prime}$ to $\boldsymbol{M U}_{0}$ are then related by (4.10) and (4.11) for some $v \in U$ ( $\boldsymbol{O H}_{0}$ ) and $\mathrm{h}=\mathrm{h}^{*} \subseteq \operatorname{\gamma rr}_{0}$.

Actually, the only penalty for dropping the hypothesis that $\alpha$ and $\alpha^{\prime}$ are weakly continuous in the above theorem is that the $C^{*}$-algebra $m_{0}$ need no longer be weakly dense in 801 .

\section{Simple $C^{\star}$-algebras}

One can reasonably argue that a perturbed dynamics should be considered as a 1 -parameter group of automorphisms of a $C^{*}$-algebra $O$, the algebra of local observables, rather than of the global von Neumann algebra M. From a mathematical point of view, it would be most natural to consider a strongly continuous 1 -parameter group of automorphisms of $\boldsymbol{Q}$. In the situations considered in physics this is not necessarily true unless one takes special care in the choice of $\boldsymbol{a}$. We shall thus suppose that $\boldsymbol{\alpha}$ is a weakly dense $c^{\star}$-subalgebra of a von Neumann algebra $\mathcal{M}$ and that $\alpha$ extends by continuity to a weakly continuous 1 -parameter group $\bar{\alpha}$ of automorphisms of $\boldsymbol{W}$. This is equivalent to assuming that $\{\boldsymbol{Q}, \alpha\}$ admits a faithful covariant representation and this is always the case when $\alpha$ is a strongly continuous 1-parameter group of automorphisms of $\boldsymbol{a}$.

We define a bounded perturbation $\alpha^{\prime}$ of $\alpha$ as in Definition 2.1 but replacing $\boldsymbol{H}$ by $\boldsymbol{Q}$. Setting $\gamma_{t}=\alpha_{t}^{\prime} \alpha_{t}^{-1}$ as before, we deduce from [8; Theorem 7$]$ that $\gamma_{t}$ may be implemented by a unitary $U_{t} \in U(\%)$. It follows that $\alpha^{\prime}$ extends by continuity to give a weakly continuous 1-parameter group $\bar{\alpha}^{\prime}$ of automorphisms of $\boldsymbol{\text { or }}$. Furthermore, by applying the Kaplansky density theorem, we deduce that $\left\|\bar{\alpha}_{t}^{\prime}-\bar{\alpha}_{t}\right\|=\left\|\alpha_{t}^{\prime}-\alpha_{t}\right\|$ and hence that $\bar{\alpha}^{\prime}$ is a bounded perturbation of $\bar{\alpha}$. We have now shown 


\subsection{Proposition}

The bounded perturbations $\alpha^{\prime}$ of $\alpha$ are in 1-1 correspondence with those bounded perturbations $\bar{\alpha}^{\prime}$ of $\bar{\alpha}$ which leave $\boldsymbol{Q}$ invariant.

This reduction of the $C^{*}$-algebra case to the von Neumann algebra case is not entirely satisfactory because although we know that the infinitesimal generators $\delta$ and $\delta^{\prime}$ of $\alpha$ and $\alpha^{\prime}$ are related by (4.10) and (4.11) for some $(h, v) \in J \boldsymbol{J}\left(\boldsymbol{g r} \boldsymbol{r}_{0}\right)$, we have no explicit characterization of those elements of $\boldsymbol{J} \boldsymbol{U}\left(\boldsymbol{\nabla} \boldsymbol{r}_{0}\right)$ which give perturbations leaving the subalgebra $\boldsymbol{Q}$ invariant.

If $\boldsymbol{Q}$ is a simple $c^{*}$-algebra with identity we may get a more precise result. This is an important case for physics because both the CCR algebra and the CAR algebra are simple. Furthermore, Borchers has shown [16] that a local net obeying certain standard assumptions of quantum field theory automatically gives rise to a simple $C^{*}$-algebra. In all these cases we may assert that $\gamma_{t}=$ ad $U_{t}$ with $U_{t} \in \boldsymbol{a}\left[7\right.$; Theorem 4.1.19]. $U_{t}$ is unique up to an element of the centre of $\boldsymbol{a}$, i.e. since $\boldsymbol{\alpha}$ is simple, up to a phase factor. We then have an exact sequence of groups

$$
1 \longrightarrow T \longrightarrow u(\alpha) \longrightarrow \operatorname{In}(\alpha) \longrightarrow 1
$$

and the cocycle $\gamma$ is a norm continuous cocycle with values in In $\boldsymbol{Q}$ (compare Proposition 2.2).

\subsection{Lemma}

Let $\boldsymbol{O}$ be a simple $c^{*}$-algebra with identity. Then $\boldsymbol{U}(\boldsymbol{G}) / \boldsymbol{T}$ may be identified with In $Q$ as topological groups in the norm topology.

Proof Pick an irreducible representation of $\boldsymbol{a}$; this is faithful so we may suppose that $\boldsymbol{O}$ is a weakly dense subalgebra of a type I factor $\boldsymbol{M}$. Arguing as before, In $Q$ may be regarded as a closed subgroup of In wr. The result now follows from Proposition 2.3.

We may now prove the analogue of Proposition 4.1 and characterize bounded perturbations in terms of 1 -cocycles with values in $\boldsymbol{U}_{\left(\boldsymbol{Q}_{0}\right)}$ where $\boldsymbol{Q}_{0}$ is the $c^{*-}$ subalgebra of elements with norm continuous orbits

$$
a_{0}=\left\{A \in a:\left\|\alpha_{t}(A)-A\right\| \rightarrow 0 \text { as } t \rightarrow 0\right\} \text {. }
$$

\subsection{Proposition}

Let $\mathcal{Q}$ be a simple $c^{*}$-algebra with identity then $\alpha^{\prime}$ is a bounded perturbation of $\alpha$ if and only if there exists a $\Gamma \in z_{\alpha}^{1}\left(\mathbb{R}, \boldsymbol{u}\left(\boldsymbol{Q}_{0}\right)\right)$ such that $\alpha_{t}^{\prime}=\operatorname{ad} \Gamma_{t} \alpha_{t}$. Proof Applying Proposition 2.6 with $\mathcal{L}=c \cdot I$ and $\omega$ an $\alpha$-invariant state of $\boldsymbol{\alpha}$, we get a continuous local cross-section for $U(\boldsymbol{C}) \rightarrow$ In $\boldsymbol{Q}$ which commutes with 
the action of $\mathbb{R}$. Arguing as in Proposition 2.8, we get a norm continuous mapping $(s, t) \rightarrow \alpha_{s}\left(U_{t}\right)$ with values in $U\left(a_{0}\right)$ such that $\alpha_{t}^{\prime}=\operatorname{adu}_{t} \alpha_{t}, t \in \mathbb{R}$. Defining $\varepsilon(s, t) I=U_{s} \alpha_{s}\left(U_{t}\right) U_{s+t}^{-1}$ we may verify as in Proposition 3.1 that $z$ is a 2 -cocycle with values in $\mathbb{T}$. Since $H^{2}(\mathbb{R}, \mathbb{T})$ is trivial, there is a continuous function $\lambda$ : $\boldsymbol{R} \rightarrow \boldsymbol{T}$ such that $z(s, t)=\lambda_{s+t} \lambda_{s}^{-1} \lambda_{t}^{-1}$ and setting $\Gamma_{s}=\lambda_{s} U_{s} \cdot \Gamma$ is a norm continuous 1 -cocycle with values in $\boldsymbol{U}\left(\boldsymbol{Q}_{0}\right)$ such that $\alpha_{t}^{\prime}=\operatorname{ad} \Gamma_{t} \alpha_{t}, t \in \mathbb{R} . \mathbf{\square}$

The analogues of Theorems 4.3 and 4.8 hold without modification since the smoothing process leading to Lemma 4.2 does not take us outside $\boldsymbol{a}_{0}$. It suffices to state the analogue of Theorem 4.8 .

\subsection{Theorem}

Let $\alpha$ be a 1-parameter automorphism group of a simple $C^{*}$-algebra with identity which may be implemented in some representation by a continuous 1-parameter unitary group. Then $\alpha^{\prime}$ is a bounded perturbation of $\alpha$ if, and only if, $\alpha$ and $\alpha^{\prime}$ lie on the same orbit under $J U\left(a_{0}\right)$. Their infinitesimal generators $\delta$ and $\delta^{\prime}$ are then related by $D\left(\delta^{\prime}\right)=V D(\delta) V^{-1}$

$$
\delta^{\prime}(A)=V \delta\left(V^{-1} A V\right) V^{-1}+[i h, A], A \in D\left(\delta^{\prime}\right)
$$

for some $v \in U\left(a_{0}\right)$ and $h=h^{*} \in \boldsymbol{Q}_{0}$. 


\section{References}

1) R. Kubo, Statistical-Mechanical Theory of Irreversible Processes I, J. Phys. Soc. Japan, 12, 570-586 (1957).

2) P.C. Martin and J. Schwinger, Theory of Many Particle Systems I, Phys. Rev. 115, 1342-1373 (1959).

3) R. Haag, D. Kastler and E.B. Trych-Pohlmeyer, Stability and Equilibrium States, Commun. Math. Phys. 38, 173-193 (1974).

4) D. Kastler, Equilibrium States of Matter and Operator Algebras

5) D.W. Robinson, Return to Equilibrium, Commun. Math. Phys. 21, 171-189 (1973).

6) H. Araki, Expansional in Banach Algebras, Ann. Scient. Ec. Norm. Sup. $\underline{6}$, (1973).

7) S. Sakai, $C^{*}$-Algebras and $W^{*}$-Algebras, Springer, Berlin Heidelberg New York (1971).

8) R.V. Kadison and J.R. Ringrose, Derivations and Automorphisms of Operator Algebras, Commun. Math. Phys. 4, 32-63 (1967).

9) A. Connes, Une Classification des facteurs de type III, Ann. Scient. Ec. Norm. Sup. 6, 133-252 (1973).

10) E.P. Wigner, On Unitary Representations of the Inhomogeneous Lorentz Group, Ann. Math. 40, 149-204 (1939).

11) R.V. Kadison and I.M. Singer, Extensions of Pure States, Amer. J. Math. 81 , 383-400 (1959).

12) R.V. Kadison, The Trace in Finite Operator Algebras, Proc. Amer. Math. Soc. $\underline{12}, 973-977(1961)$.

13) Dunford and J.T. Schwartz, Linear Operators, Vo1. I Interscience, New York (1958).

14) K. Iwasawa, One some types of Topological Groups, Ann. Math. 50, 507-558 (1949).

15) E. Hille and R.S. Phillips, Functional Analysis and Semigroups, AMS, Providence N.Y. (1957).

16) H.J. Borchers, A Remark on a Theorem of B. Misra, Commun. Math. Phys. 4, 315-323 (1967). 